\title{
Antibacterial Activities and Synergistic Interaction of Citrus Essential Oils and Limonene with Gentamicin against Clinically Isolated Methicillin-Resistant Staphylococcus aureus
}

\author{
Apichai Sreepian $\mathbb{D}^{1},{ }^{1}$ Supaluk Popruk $\left(\mathbb{D},{ }^{2}\right.$ Daranee Nutalai $\mathbb{D}^{3},{ }^{3}$ Chayanit Phutthanu $\left(\mathbb{D},{ }^{3}\right.$ \\ and Preeyaporn M. Sreepian $\left(^{1}{ }^{1}\right.$ \\ ${ }^{1}$ Faculty of Medical Technology, Rangsit University, Pathum Thani 12000, Thailand \\ ${ }^{2}$ Department of Protozoology, Faculty of Tropical Medicine, Mahidol University, Bangkok 10400, Thailand \\ ${ }^{3}$ Division of Microbiology, Department of Central Laboratory and Blood Bank, Faculty of Medicine, Vajira Hospital, \\ Navamindradhiraj University, Bangkok 10300, Thailand
}

Correspondence should be addressed to Preeyaporn M. Sreepian; preeyaporn.m@rsu.ac.th

Received 25 November 2021; Accepted 4 January 2022; Published 28 February 2022

Academic Editor: Wongi Min

Copyright (c) 2022 Apichai Sreepian et al. This is an open access article distributed under the Creative Commons Attribution License, which permits unrestricted use, distribution, and reproduction in any medium, provided the original work is properly cited.

\begin{abstract}
Citrus reticulata Blanco and Citrus aurantifolia are the edible plants which contain several biological properties including antibacterial activity. The aims of the present study were to determine the chemical compositions and evaluate antibacterial activities of citrus essential oils extracted from the fruit peels of $C$. reticulata (CREO) and C. aurantifolia (CAEO), alone and in combination with gentamicin, against a panel of clinically isolated methicillin-resistant S. aureus (MRSA) $(n=40)$ and methicillinsusceptible $S$. aureus (MSSA) $(n=45)$. Gas chromatography-mass spectrometry analysis revealed that 12 and 25 compounds were identified in CREO and CAEO with the most predominant compound of limonene (62.9-72.5\%). The antibacterial activities were determined by agar disk diffusion and resazurin-based microdilution methods. The results found that almost all MRSA isolates were resistant to ciprofloxacin, erythromycin, and clindamycin, and some isolates were resistant to gentamicin. CREO and CAEO exhibited inhibitory effects toward clinical isolates (MIC: $1.0-32.0$ and $8.0-32.0 \mathrm{mg} / \mathrm{mL}$, respectively), with a similar trend to limonene (MIC: $1.0-32.0 \mathrm{mg} / \mathrm{mL}$ ). However, the higher antibacterial effects were found in CREO and limonene when compared to CAEO $(p<0.01)$. In combination effect, the results showed the synergistic interaction of gentamicin with CREO and limonene on the MRSA and MSSA isolates (FIC indexes: $0.012-0.258$ and $0.012-0.375$ ), but that interaction of gentamicin with CAEO was observed only on MRSA (FIC index: 0.012-0.016). These findings demonstrated the potential of these citrus essential oils as natural antibacterial agents that may contribute to reduce the emerging of antimicrobial-resistant bacteria.
\end{abstract}

\section{Introduction}

Staphylococcus aureus, a Gram-positive commensal bacterium, is mainly colonized in the nasal carriage as well as in the skin, axillae, perineum, and pharynx [1]. It can cause a wide variety of infectious diseases, ranging from mild skin and soft tissue infections to life-threatening such as endocarditis, osteomyelitis, pneumonia, and bacteremia. Due to overuse of antibiotics, the emergence of the antibiotic-resistant phenotype has been reported worldwide. Methicillinresistant $S$. aureus (MRSA), caused by the acquisition of staphylococcal cassette chromosome mec (SCCmec) which carries the mecA gene that is responsible for the production of an altered penicillin-binding protein $2 \mathrm{a}$ (PBP2a), thus associated with decreased affinity for all $\beta$-lactam antibiotics. The relative high burden of hospital-acquired MRSA (HAMRSA) and community-acquired MRSA (CA-MRSA) is a major concern worldwide. HA-MRSA has a higher mortality rate, an increased duration of hospitalization, and a higher healthcare cost [2]. Vancomycin has been the drug of choice and is currently noticed as the last resort for the treatment of severe MRSA infection [2,3]. However, nephrotoxicity, 
hypotension, and hypersensitivity reactions are commonly presented; thus, drug monitoring is required [4]. In this context, the searching for a new candidate of alternative anti-MRSA agent with a lesser toxicity is required, and one of the possible ways to support this goal is the use of plantderived agents.

The herbal medicinal products have been considered as a natural source for alternative treatment for bacterial infections. Citrus reticulata Blanco (commonly known as mandarin orange and tangerine orange) and Citrus aurantifolia (commonly known as key lime, common lime, and lime) are edible fruits belonging to the family Rutaceae. They are cultivated in tropical and subtropical regions worldwide. Generally, they can be applied as food and drink flavoring ingredients as they have signature citrus flavoring and scents. C. aurantifolia is traditionally used to promote the digestion process and for antidiabetic and antihypercholesterolemic purposes. The oil extracted from the C. aurantifolia fruits can be used for relieving cold, asthma, and arthritis [5]. The productivities of C. reticulata and C. aurantifolia were annually reported at 153,375 and 213,716 tons in Thailand [6]. Several biological properties of crude extracts and essential oils from Citrus spp., C. reticulata and C. aurantifolia, have been reported, including antioxidant [7-11], antibiofilm [12], antibacterial, and antifungal activities $[10,13]$. The dried peel and pulp extracts of C. aurantifolia from Indonesia possessed antioxidant, antidiabetic, and antibacterial activities against S. aureus and K. pneumoniae [9].

Additionally, antibacterial activities of crude extracts and essential oils (EOs) from different parts (leaves, stems, roots, flowers, and peels) of C. reticulata and C. aurantifolia have demonstrated broad-spectrum antibacterial activities toward clinically important pathogens with a major activity against Gram-positive bacteria, especially $S$. aureus $[7,9-11,14-20]$. Previously, some studies have investigated the antistaphylococcal effect of these citrus-derived products, mostly against the reference strains of MRSA and methicillin-susceptible $S$. aureus (MSSA). Bektaš et al. reported the inhibitory effect of orange juices on MSSA ATCC 25923, MRSA NCTC 1249, and clinical isolates of MRSA [21]. Vong et al. reported the inhibitory effect of the fruit juice extract of C. aurantifolia from Malaysia against MRSA ATCC 33591 [22]. Chao et al. reported the inhibitory effects of several commercial citrus EOs including $C$. aurantifolia EO which inhibited the growth of MRSA ATCC 700699 [23]. Up to now, there are limited data on anti-MRSA effects of the citrus extracts and EOs toward the clinical isolates, in which more variation in the antimicrobial susceptibility pattern is observed. Therefore, this study aimed to determine the antibacterial activities of the citrus EOs and their major compounds against clinically isolated MRSA and MSSA. In addition, the synergistic effects of citrus EOs and their major compounds to improve the efficacy of the antibacterial agent, gentamicin, against clinically isolated MRSA and MSSA were also investigated.
TABLE 1: Clinical sources of tested bacteria used in this study.

\begin{tabular}{lcc}
\hline Sources & \multicolumn{2}{c}{ No. (\%) } \\
& MRSA & MSSA \\
\hline Pus/wound & $9(22.5)$ & $16(35.5)$ \\
Hemoculture & $8(20.0)$ & $13(28.9)$ \\
Sputum & $17(42.5)$ & $13(28.9)$ \\
Urine/stool & $6(15.0)$ & $3(6.7)$ \\
\hline Total & $40(100)$ & $45(100)$ \\
\hline
\end{tabular}

\section{Materials and Methods}

2.1. Plant Materials. The fresh fruits of C. reticulata Blanco (mandarin orange) and C. aurantifolia (Christm.) Swingle (lime) were collected from Chiang Rai Province, the northernmost of Thailand, in December 2019. The plant samples were identified, and voucher specimens (BCU no. 015859 and BCU no. 015858) were housed at the herbarium of the Department of Botany, Faculty of Science, Chulalongkorn University, Thailand. The fruit peels were ground, suspended in distilled water, and then processed through hydrodistillation for $3 \mathrm{~h}$. Essential oils were separated from the aqueous layer using a micropipette. The oils were dried over anhydrous sodium sulfate, filtered, and stored at $4^{\circ} \mathrm{C}$ [24]. In this study, the percentage yields of extracted C. reticulata essential oil (CREO) and C. aurantifolia essential oil (CAEO) were $0.48 \%$ and $0.30 \%$, respectively. The extracted EOs with a density of $0.8 \mathrm{~g} / \mathrm{mL}$ were stored at $4^{\circ} \mathrm{C}$ and protected from light. A stock solution was prepared at the concentration of $400 \mathrm{mg} / \mathrm{mL}$ in dimethyl sulfoxide (DMSO) before use.

2.2. Bacterial Organisms. The tested bacterial organisms contained 2 American Type Culture Collection (ATCC) bacterial strains including Staphylococcus aureus ATCC 43300 (methicillin-resistant S. aureus, MRSA) and S. aureus ATCC 25923 (methicillin-susceptible $S$. aureus, MSSA) as well as clinically isolated MRSA $(n=40)$ and MSSA $(n=45)$. The clinical isolates were obtained from the Division of Microbiology, Department of Central Laboratory and Blood Bank, Faculty of Medicine, Vajira Hospital, Navamindradhiraj University, Thailand, and identified by matrixassisted laser desorption/ionization time-of-flight mass spectrometry (MALDI-TOF MS). These clinical isolates were originally collected from skin, bloodstream, respiratory, and urinary tracts (Table 1). The bacteria were maintained at $-70^{\circ} \mathrm{C}$ and subcultured on blood agar at $37^{\circ} \mathrm{C}$ overnight prior the assay.

2.3. Antibiotic Susceptibility Test. The susceptibilities of 8 antibiotics (Oxoid, England) including cefoxitin $(30 \mu \mathrm{g})$, ampicillin $(10 \mu \mathrm{g})$, amoxicillin $(30 \mu \mathrm{g})$, gentamicin $(10 \mu \mathrm{g})$, ciprofloxacin $(5 \mu \mathrm{g})$, erythromycin $(5 \mu \mathrm{g})$, clindamycin $(2 \mu \mathrm{g})$, and vancomycin (E-test) were characterized by agar diffusion, according to CLSI (2019). The susceptibility patterns were interpreted by the inhibition zone diameter 
(IZD). S. aureus was considered as MRSA when IZD of cefoxitin $\leq 21 \mathrm{~mm}$ and MSSA when IZD $\geq 22 \mathrm{~mm}$ [25].

2.4. GC-MS Analysis. The separation and identification of volatile components of CREO and CAEO were carried out by gas chromatography-mass spectrometry (GC-MS) (GC 7890A/MS 5975C-MSD; Agilent Technologies, CA, USA). The capillary column Mega-5MS $(30 \mathrm{~m} \times 0.25 \mathrm{~mm} \times 0.25 \mu \mathrm{m})$ was used. The GC conditions were programmed as the injection temperature $250^{\circ} \mathrm{C}$, with oven temperature initially set at $50^{\circ} \mathrm{C}$ for $1 \mathrm{~min}$ and then gradually increasing at the rate of $3^{\circ} \mathrm{C} / \mathrm{min}$ up to $250^{\circ} \mathrm{C}$ and held for $5 \mathrm{~min}$. Helium was used as the carrier gas with a constant flow rate of $1.0 \mathrm{~mL} / \mathrm{min}$. The volume of injection was $1 \mu \mathrm{L}$ of ethanol solution in a split mode $(1: 10)$. The MS transfer line temperature was set at $250^{\circ} \mathrm{C}$ with the electron ionization (EI) mode at $70 \mathrm{eV}$ ionization potential. The mass-to-charge $(\mathrm{m} / \mathrm{z})$ range was from 40 to $650 \mathrm{~m} / \mathrm{z}$. Compounds were further identified by matching their mass spectra fragmentation pattern and retention time with standard reference compounds, compared their MS results with the NIST 2011 library, and stored in the GC/MS database for confirmation.

2.5. Agar Disk Diffusion. Agar disk diffusion was performed to screen the in vitro antibacterial activities of the EOs as previously described [26]. Sterilized disks $(6 \mathrm{~mm}$ in diameter) impregnated with $10 \mu \mathrm{L}$ of each $\mathrm{EO}$ or pure limonene (Lot no. MKCD9298; Sigma-Aldrich, St. Louis, MO, USA) were placed on the surface of the Mueller-Hinton agar (MHA; Oxoid, England) plate after tested bacteria (0.5 McFarland unit) were inoculated. The disk containing $10 \mu \mathrm{L}$ of $4 \%$ DMSO and commercial gentamicin disk $(10 \mu \mathrm{g})$ (Oxoid, England) were used as negative and positive controls, respectively. After incubation at $37^{\circ} \mathrm{C}$ for $18-24 \mathrm{~h}$, the IZD of the EOs was measured and interpreted following the criteria: no activity, IZD $=6 \mathrm{~mm}$; weak activity, $6 \mathrm{~mm}<\mathrm{IZD} \leq 12 \mathrm{~mm}$; moderate activity, $12 \mathrm{~mm}<\mathrm{IZD}<20 \mathrm{~mm}$; and strong activity, IZD $>20 \mathrm{~mm}$ [27].

2.6. Determination of MIC. The minimum inhibitory concentrations (MICs) of the EOs were evaluated by the resazurinbased 96-well plate microdilution method as previously described with some modifications [28]. Fifty microliters of various concentrations of EOs and limonene were prepared by a serial two-fold dilution with cation-adjusted Mueller-Hinton broth (CAMHB) in a sterile 96-well microplate to obtain the final concentrations ranging from 0.1 to $32.0 \mathrm{mg} / \mathrm{mL}$. In addition, gentamicin supplement was included in the experiment with the final concentration of 0.1 to $256.0 \mu \mathrm{g} / \mathrm{mL}$. Afterward, $50 \mu \mathrm{L}$ of tested bacteria was added into each well to obtain a final concentration of $5 \times 10^{5} \mathrm{CFU} / \mathrm{mL}$. Only EO dissolved in CAMHB (oil control), only bacterial suspension in CAMHB (bacterial control), and 4\% DMSO with bacterial suspension (diluent control) were also included. After incubation at $37^{\circ} \mathrm{C}$ for $24 \mathrm{~h}, 5 \mu \mathrm{L}$ of $0.015 \%$ resazurin (Sigma-Aldrich, St. Louis, MO, USA) was added into each well and further incubated for $2 \mathrm{~h}$ in the dark. The bacterial growth was visually observed by the change of resazurin natural color (blue-purple) into the reduced form (red-colorless). The MIC was defined by the lowest concentration that completely inhibits the growth of bacteria (no color change). Consequently, one loop of the MIC suspension that showed no color change was cultivated on the MHA plate and further incubated at $37^{\circ} \mathrm{C}$ for $18-24 \mathrm{~h}$. The minimum bactericidal concentration (MBC) was defined by the lowest concentration that completely kills bacteria on the agar plate. The MIC index (MBC/MIC ratio) was calculated to classify the type of antimicrobial substances and interpreted using the following criteria: bactericidal, MIC index $\leq 4$; bacteriostatic, MIC index $>4$; and resistance, MIC index $\geq 32$ [29].

2.7. Checkerboard Titration Assay. The checkerboard titration assay was performed to evaluate the synergistic interaction among EOs and limonene combined with gentamicin against 7 clinical isolates. This method was based on the broth microdilution assay with the final volume of $100 \mu \mathrm{L}$. In brief, $25 \mu \mathrm{L}$ of various concentrations of EOs or limonene ranging from 0.3 to $32.0 \mathrm{mg} / \mathrm{mL}$ was prepared by serial 2-fold dilution in the 96-well microplate. In the meanwhile, various concentrations of gentamicin were prepared ranging from 0.001 to $128.0 \mu \mathrm{g} / \mathrm{mL}$. Then, $25 \mu \mathrm{L}$ of each concentration of gentamicin was added into each concentration of $\mathrm{EO}$ or limonene to perform checkerboard testing. Fifty microliters of tested bacteria were added into each well to obtain a final concentration of $5 \times 10^{5} \mathrm{CFU} / \mathrm{mL}$ and incubated at $37^{\circ} \mathrm{C}$ for $18-24 \mathrm{~h}$. Afterward, $5 \mu \mathrm{L}$ of $0.015 \%$ resazurin was added and further incubated for $2 \mathrm{~h}$ in the dark. The bacterial growth was visually observed by the color change of resazurin. The combination effect of either EOs or limonene with gentamicin was determined by using the fractional inhibitory concentration index (FICI) value using the following formula:

$\mathrm{FIC}=\frac{\text { MIC of the EOs, limonene or gentamicin in combination }}{\text { MIC of the EOs, limonene or gentamicin alone }}$

FICI $=$ FIC of the EOs or limonene + FIC of gentamicin

The interaction was interpreted by using the following criteria: $\quad \mathrm{FICI} \leq 0.5, \quad$ synergy; $0.5<\mathrm{FICI} \leq 1, \quad$ additive; $1<\mathrm{FICI} \leq 4$, indifference; and FICI $>4$, antagonism [30].

2.8. Statistical Analysis. All experiments were performed in triplicate. The data were analyzed with the descriptive statistics, Kruskal-Wallis test, and Mann-Whitney $U$ test using the IBM Statistical Package for Social Services (SPSS) version 21.0 (IBM, Armonk, NY, USA). A $p$ value $<0.05$ was considered statistically significant.

\section{Results}

3.1. Chemical Composition of EOs. The wide variety of chemical compositions of the citrus EOs, Citrus reticulata (CREO) and Citrus aurantifolia (CAEO), are presented in Tables 2 and 3, and the GC-MS chromatograms are shown in 
TABLE 2: Chemical compositions of the Citrus reticulata Blanco essential oil.

\begin{tabular}{|c|c|c|c|c|c|}
\hline No. & Compounds & Retention time (min) & Retention index* & Composition (\%) & Quality \\
\hline 1 & $\alpha$-Pinene & 8.383 & 936 & 0.45 & 97 \\
\hline 2 & Sabinene & 9.549 & 966 & 0.04 & 94 \\
\hline 3 & $\beta$-Pinene & 9.776 & 972 & 0.12 & 97 \\
\hline 4 & $\beta$-Myrcene & 10.562 & 992 & 1.03 & 91 \\
\hline 5 & D-Limonene & 12.575 & 1039 & 72.53 & 99 \\
\hline 6 & $\beta$-Linalool & 15.768 & 1111 & 0.34 & 83 \\
\hline 7 & Terpinen-4-ol & 19.567 & 1193 & 0.04 & 94 \\
\hline 8 & $\alpha$-Terpineol & 20.422 & 1212 & 0.18 & 90 \\
\hline 9 & Carvone & 22.532 & 1258 & 0.03 & 94 \\
\hline 10 & Perillal & 24.015 & 1291 & 0.02 & 90 \\
\hline 11 & $(-)-\beta$-Elemene & 28.504 & 1394 & 0.04 & 90 \\
\hline 12 & D-Germacrene & 32.434 & 1488 & 0.01 & 96 \\
\hline
\end{tabular}

* Retention index relative to $n$-alkanes (C8-C40) on the Mega-5MS column. The bold values indicate the representative data of the major compound.

TABle 3: Chemical compositions of the Citrus aurantifolia essential oil.

\begin{tabular}{|c|c|c|c|c|c|}
\hline No. & Compounds & Retention time (min) & Retention index* & Composition (\%) & Quality \\
\hline 1 & Thujene & 8.087 & 928 & 0.45 & 91 \\
\hline 2 & $\alpha$-Pinene & 8.390 & 936 & 1.55 & 98 \\
\hline 3 & Camphene & 9.059 & 953 & 0.04 & 96 \\
\hline 4 & Sabinene & 9.548 & 966 & 0.29 & 91 \\
\hline 5 & $\beta$-Pinene & 9.790 & 972 & 11.41 & 97 \\
\hline 6 & $\beta$-Myrcene & 10.569 & 992 & 0.92 & 91 \\
\hline 7 & $\alpha$-Phellandrene & 11.369 & 1012 & 0.04 & 91 \\
\hline 8 & $\alpha$-Terpinene & 11.824 & 1022 & 0.23 & 96 \\
\hline 9 & D-Limonene & 12.575 & 1039 & 62.95 & 98 \\
\hline 10 & $\gamma$-Terpinene & 13.741 & 1065 & 13.30 & 96 \\
\hline 11 & Terpinolene & 14.858 & 1091 & 0.43 & 97 \\
\hline 12 & $\beta$-Linalool & 15.829 & 1111 & 0.19 & 80 \\
\hline 13 & Terpinen-4-ol & 19.581 & 1194 & 0.24 & 93 \\
\hline 14 & $\alpha$-Terpineol & 20.422 & 1212 & 0.45 & 72 \\
\hline 15 & cis-Geraniol & 21.532 & 1236 & 0.26 & 93 \\
\hline 16 & $\beta$-Citral & 22.091 & 1249 & 0.55 & 87 \\
\hline 17 & $\alpha$-Citral & 23.47 & 1279 & 0.74 & 97 \\
\hline 18 & $\delta$-Elemene & 26.042 & 1337 & 0.06 & 98 \\
\hline 19 & $\alpha$-Bergamotene & 29.435 & 1416 & 0.07 & 98 \\
\hline 20 & Caryophyllene & 29.821 & 1425 & 0.39 & 99 \\
\hline 21 & $\alpha$-trans-Bergamotene & 30.255 & 1436 & 0.80 & 91 \\
\hline 22 & Terpinolene & 14.858 & 1091 & 0.43 & 97 \\
\hline 23 & $\beta$-Linalool & 15.829 & 1111 & 0.19 & 80 \\
\hline 24 & Terpinen-4-ol & 19.581 & 1194 & 0.24 & 93 \\
\hline 25 & $\alpha$-Terpineol & 20.422 & 1212 & 0.45 & 72 \\
\hline
\end{tabular}

* Retention index relative to $n$-alkanes (C8-C40) on the Mega-5MS column. The bold values indicate the representative data of the major compound.

Figures 1 and 2. Twelve and 25 compounds accounted for $74.8 \%$ of the total CREO and $96.8 \%$ of the total CAEO, respectively, with predominant monoterpene hydrocarbons (74.2\% and $91.6 \%)$, followed by oxygenated monoterpenes $(0.6 \%$ and $2.4 \%)$ and sesquiterpene hydrocarbons $(0.1 \%$ and $2.8 \%)$.

In CREO, D-limonene (72.53\%) was the major component of monoterpene hydrocarbons followed by $\beta$-myrcene $(1.03 \%)$ and $\alpha$-pinene $(0.45 \%)$, while $\beta$-linalool $(0.34 \%)$ and $\alpha$-terpineol $(0.18 \%)$ were the major components of oxygenated monoterpenes. The compositions of remaining 7 compounds in CREO ranged from 0.01 to $0.12 \%$ (Table 2). In CAEO, D-limonene (62.95\%) was the major component of monoterpene hydrocarbons followed by $\gamma$-terpinene $(13.30 \%), \beta$-pinene $(11.40 \%), \alpha$-pinene
(1.55\%), $\beta$-myrcene $(0.92 \%)$, and $\alpha$-thujene/ $\beta$-thujene $(0.45 \%)$, while $\alpha$-citral $(0.74 \%), \beta$-citral $(0.55 \%)$, and $\alpha$-terpineol $(0.45 \%)$ were the major components of oxygenated monoterpenes; and $\beta$-bisabolene $(1.13 \%), \alpha$-transbergamotene $(0.80 \%)$, and caryophyllene $(0.39 \%)$ were the major components of sesquiterpene hydrocarbons. The compositions of remaining 13 compounds in CAEO ranged from 0.04 to $0.43 \%$ (Table 3 ).

3.2. Antibiotic Susceptibility Pattern. The susceptibility patterns of tested bacteria in this study are shown in Table 4. The susceptibility to cefoxitin $(10 \mu \mathrm{g})$ was used to classify between MRSA and MSSA. The results showed that $S$. aureus ATCC 43300 and 40 clinical isolates of $S$. aureus were 


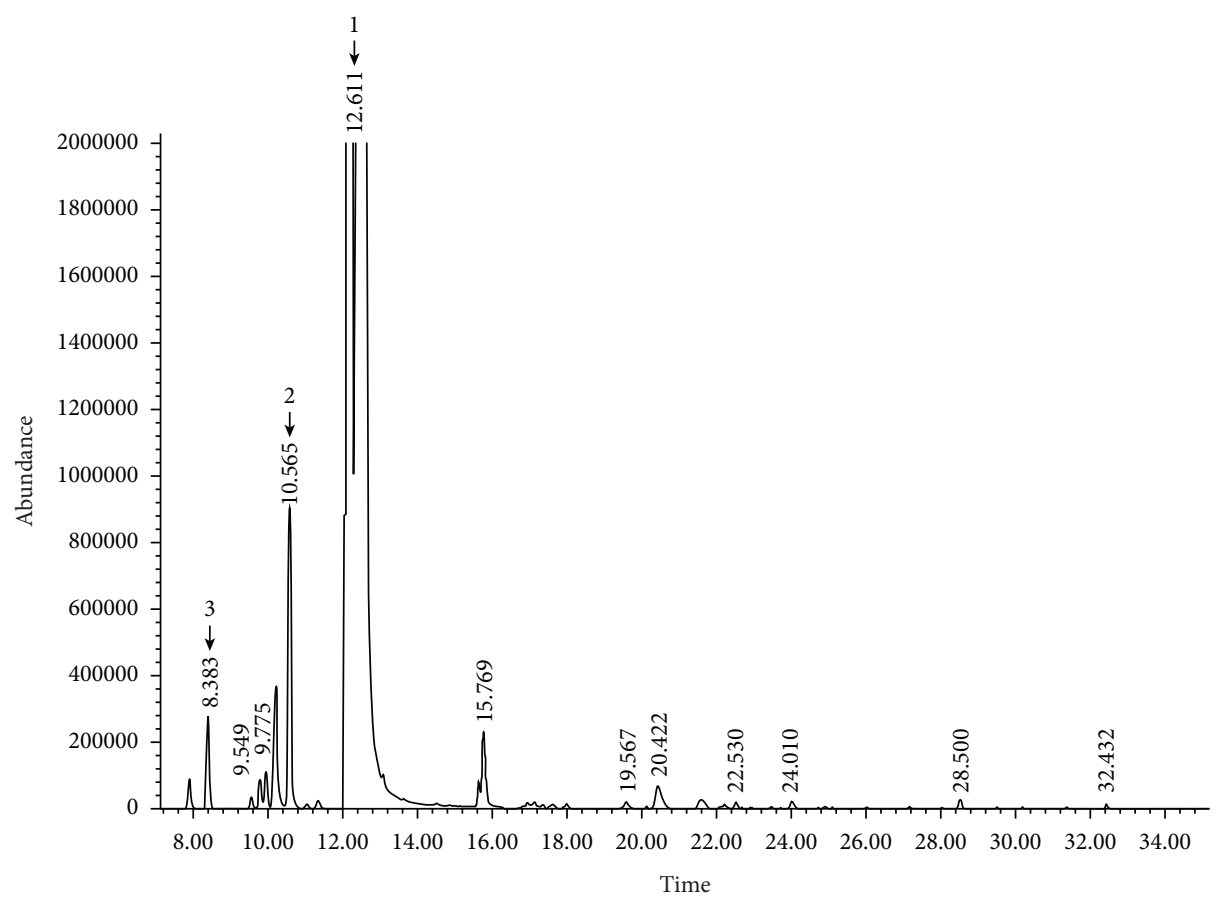

Figure 1: Representative GC-MS chromatogram of the Citrus reticulata Blanco essential oil. Major compound peaks were marked as follows: D-limonene (1), $\beta$-myrcene (2), and $\alpha$-pinene (3).

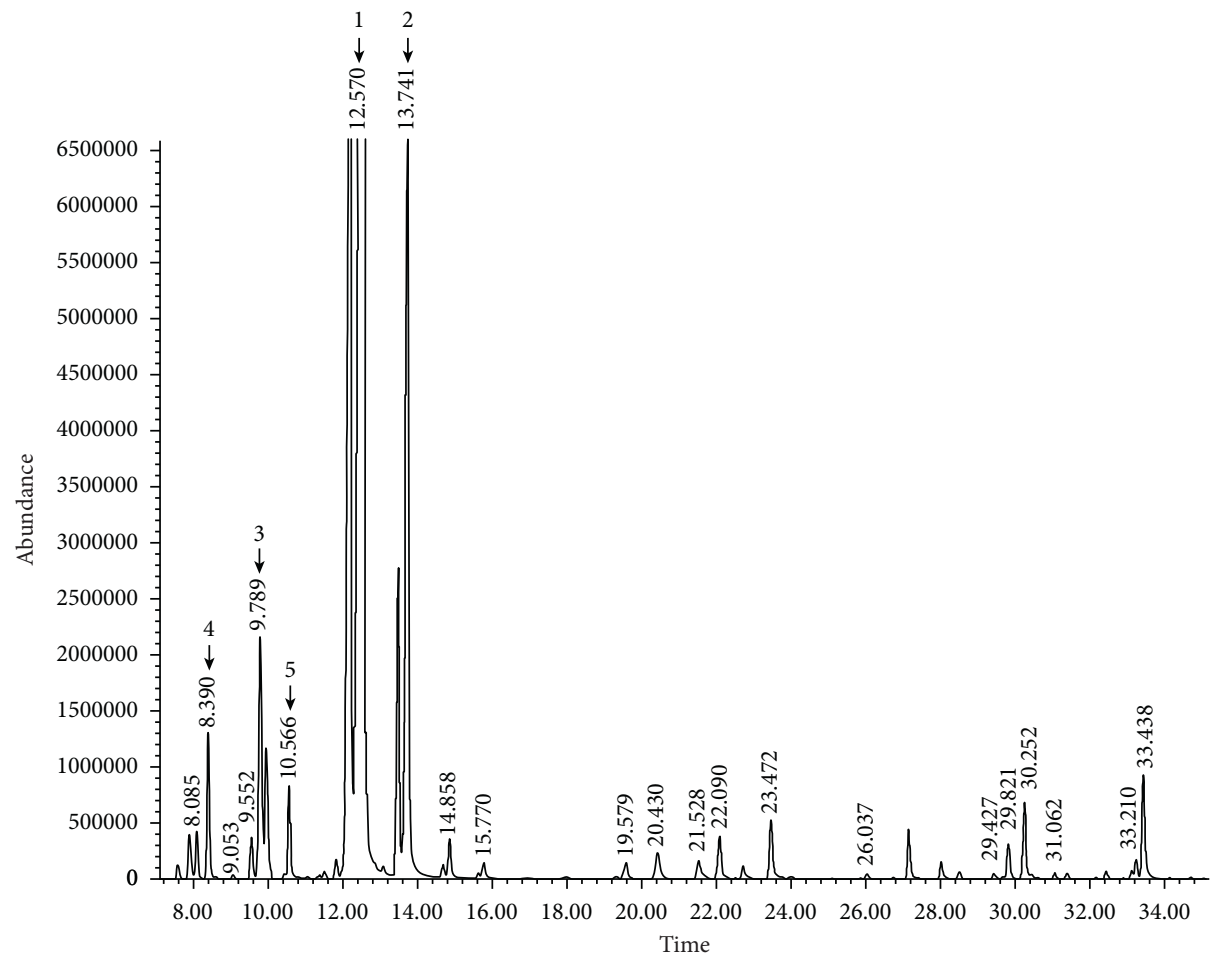

Figure 2: Representative GC-MS chromatogram of the Citrus aurantifolia essential oil. Major compound peaks were marked as follows: Dlimonene (1), $\gamma$-terpinene (2), $\beta$-pinene (3), $\alpha$-pinene (4), and $\beta$-myrcene (5).

resistant to cefoxitin (IZD: $14.0 \pm 1.7 \mathrm{~mm}$ and $7.2 \pm 2.4 \mathrm{~mm}$, respectively), indicating MRSA phenotype, while $S$. aureus ATCC 25923 and the other 45 clinical isolates were susceptible to cefoxitin (IZD: $28.7 \pm 5.0 \mathrm{~mm}$ and $30.0 \pm 2.3 \mathrm{~mm}$, respectively), indicating MSSA phenotype.
It also showed that MSSA ATCC 25923 was susceptible to all other tested antibiotics including gentamicin (IZD: $26.7 \pm 1.2 \mathrm{~mm}$ ), ciprofloxacin (IZD: $25.0 \pm 1.0 \mathrm{~mm}$ ), erythromycin (IZD: $28.0 \pm 1.0 \mathrm{~mm}$ ), clindamycin (IZD: $30.0 \pm 2.1 \mathrm{~mm}$ ), and vancomycin (MIC: $1.9 \pm 0.1 \mu \mathrm{g} / \mathrm{mL}$ ), 
TABLE 4: Antibiotic susceptibility pattern of tested bacteria.

\begin{tabular}{|c|c|c|c|c|c|c|c|c|}
\hline \multirow{2}{*}{ Bacterial strains } & \multicolumn{7}{|c|}{ IZD (mm) } & \multirow{2}{*}{$\begin{array}{c}\mathrm{MIC}^{*}(\mu \mathrm{g} / \mathrm{mL}) \\
\mathrm{VA}\end{array}$} \\
\hline & FOX & $\mathrm{CN}$ & CIP & ERY & DA & AMP & $\mathrm{AMC}$ & \\
\hline \multicolumn{9}{|l|}{ Reference strains } \\
\hline S. aureus ATCC 43300 & $14.0 \pm 1.7(\mathrm{R})$ & $23.0 \pm 1.0(\mathrm{~S})$ & $25.0 \pm 3.6(\mathrm{~S})$ & $6.0 \pm 0.0(\mathrm{R})$ & $6.0 \pm 0.0(\mathrm{R})$ & $12.0 \pm 3.5$ & $14.7 \pm 2.3$ & $1.0 \pm 0.0(\mathrm{~S})$ \\
\hline S. aureus ATCC 25923 & $28.7 \pm 5.0(\mathrm{~S})$ & $26.7 \pm 1.2(\mathrm{~S})$ & $25.0 \pm 1.0(\mathrm{~S})$ & $28.0 \pm 1.0(\mathrm{~S})$ & $30.3 \pm 2.1(\mathrm{~S})$ & $33.0 \pm 2.6$ & $30.0 \pm 5.3$ & $1.9 \pm 0.1(\mathrm{~S})$ \\
\hline MRSA isolates $(n=40)$ & $7.2 \pm 2.4^{* *}$ & $17.7 \pm 8.4^{* *}$ & $8.5 \pm 7.2^{* *}$ & $9.7 \pm 7.9^{* *}$ & $9.7 \pm 8.1^{* *}$ & $9.7 \pm 5.8^{* *}$ & $10.4 \pm 6.6^{* *}$ & $0.5 \pm 0.2$ \\
\hline No. of resistance (\%) & $40(100 \%)$ & $15(37.5 \%)$ & $38(95.0 \%)$ & $31(77.5 \%)$ & $30(76.9 \%)$ & NA & NA & $0(0 \%)$ \\
\hline No. of intermediate (\%) & $0(0 \%)$ & $0(0 \%)$ & $0(0 \%)$ & $0(0 \%)$ & $0(0 \%)$ & NA & NA & $0(0 \%)$ \\
\hline No. of sensitive (\%) & $0(0 \%)$ & $25(62.5 \%)$ & $2(5 \%)$ & $9(22.5 \%)$ & $9(23.1 \%)$ & NA & NA & $40(100 \%)$ \\
\hline MSSA isolates $(n=45)$ & $30.0 \pm 2.3$ & $22.3 \pm 3.5$ & $25.2 \pm 6.3$ & $23.0 \pm 8.6$ & $23.3 \pm 7.7$ & $21.9 \pm 8.6$ & $25.6 \pm 6.9$ & NA \\
\hline No. of resistance (\%) & $0(0 \%)$ & $2(4.4 \%)$ & $3(6.7 \%)$ & $4(16.7 \%)$ & $4(14.8 \%)$ & NA & NA & NA \\
\hline No. of intermediate (\%) & $0(0 \%)$ & $0(0 \%)$ & $3(6.7 \%)$ & $3(12.5 \%)$ & $2(7.4 \%)$ & NA & NA & NA \\
\hline No. of sensitive (\%) & $45(100 \%)$ & $43(95.6 \%)$ & $39(86.6 \%)$ & $17(70.8 \%)$ & $21(77.8 \%)$ & NA & NA & NA \\
\hline
\end{tabular}

Values are expressed as mean \pm SD of triplicate experiments. AMP: ampicillin $(10 \mu \mathrm{g})$; AMC: amoxicillin $(30 \mu \mathrm{g})$; FOX: cefoxitin $(30 \mu \mathrm{g})$; CN: gentamicin $(10 \mu \mathrm{g})$; CIP: ciprofloxacin $(5 \mu \mathrm{g})$; ERY: erythromycin $(15 \mu \mathrm{g})$; DA: clindamycin $(2 \mu \mathrm{g})$; VA: vancomycin; S: susceptible; R: resistant; NA: not applicable. Susceptibilities of $S$. aureus against AMP and AMC were not interpreted by CLSI (2019). *Vancomycin-susceptible S. aureus (VSSA), MIC $\leq 2 \mu \mathrm{g} / \mathrm{mL}$. ${ }^{* *} p<0.01$ (Mann-Whitney U test), statistically significant when compared to the MSSA isolate.

while MRSA ATCC 43300 was susceptible to gentamicin (IZD: $23.0 \pm 1.0 \mathrm{~mm}$ ), ciprofloxacin (IZD: $25.0 \pm 3.6 \mathrm{~mm}$ ), and vancomycin (MIC: $1.0 \pm 0.0 \mu \mathrm{g} / \mathrm{mL}$ ) but resistant to erythromycin (IZD: $6.0 \pm 0.0 \mathrm{~mm}$ ) and clindamycin (IZD: $6.0 \pm 0.0 \mathrm{~mm}$ ). The IZDs of ampicillin and amoxicillin were compared since there were no interpreting criteria for the susceptibility pattern of these antibiotics, and it showed that MRSA ATCC 43300 was lesser susceptible to ampicillin and amoxicillin than MSSA ATCC 25923 (IZD: $12.0 \pm 3.5 \mathrm{~mm}$ vs. $33.0 \pm 2.6 \mathrm{~mm}$ for ampicillin and $14.7 \pm 2.3 \mathrm{~mm}$ vs. $30.0 \pm 5.3 \mathrm{~mm}$ for amoxicillin).

The resistant rate to gentamicin among clinical isolates of MRSA (15/40, 37.5\%) was higher than that of MSSA (2/45, $4.4 \%)$ with the IZDs of $17.7 \pm 8.4 \mathrm{~mm}$ and $22.3 \pm 3.5 \mathrm{~mm}$, respectively $(p<0.01)$. For other antibiotics, MRSA isolates showed high rates of resistance to ciprofloxacin, erythromycin, and clindamycin (76.9-95.0\%) when compared to MSSA isolates (6.7-16.7\%) with the IZDs of $8.5 \pm 7.2 \mathrm{~mm}$, $9.7 \pm 7.9 \mathrm{~mm}$, and $9.7 \pm 8.1 \mathrm{~mm}$ for MRSA and the IZDs of $25.2 \pm 6.3 \mathrm{~mm}, 23.0 \pm 8.6 \mathrm{~mm}$, and $23.3 \pm 7.7 \mathrm{~mm}$ for MSSA $(p<0.01)$. In this study, all MRSA $(40 / 40,100 \%)$ were susceptible to vancomycin, which is a last-resort antibiotic for multidrug-resistant (MDR) bacteria at MIC $0.5 \pm 0.2 \mu \mathrm{g} /$ $\mathrm{mL}$. As the expected results, MRSA isolates were less susceptible to $\beta$-lactam antibiotics, ampicillin and amoxicillin, when compared to MSSA isolates with the IZDs of $9.7 \pm 5.8 \mathrm{~mm}$ vs. $21.9 \pm 8.6 \mathrm{~mm}$ for ampicillin and $10.4 \pm 6.6 \mathrm{~mm}$ vs. $25.6 \pm 6.9 \mathrm{~mm}$ for amoxicillin $(p<0.01)$. All these together, it demonstrated that MRSA isolates seem to be resistant to all tested antibiotics except for vancomycin.

In addition, MDR $S$. aureus, which is classified by the resistant pattern to at least one agent among at least three antibiotic classes, was found at high rate among MRSA isolates $(31 / 40,77.5 \%)$, but only one was observed among MSSA isolates (1/45, 2.2\%). This MDR MSSA isolate was resistant to all antibiotics except cefoxitin. Beside the resistance to cefoxitin $(31 / 31,100 \%)$, the most common antibiotic resistance of MDR MRSA was erythromycin (31/ $31,100 \%)$, followed by ciprofloxacin $(30 / 31,96.8 \%)$, clindamycin $(30 / 31,96.8 \%)$, and gentamicin $(15 / 31,48.4 \%)$ (data not shown).

\subsection{Antibacterial Activity of Citrus EOs and Limonene.} The values of IZD, MIC, and MBC of the citrus EOs and pure limonene against tested bacteria are shown in Table 5. In this study, sterile disk containing 4\% DMSO had no inhibitory effect, while commercial gentamicin disk $(10 \mu \mathrm{g})$ had the effect toward all $S$. aureus isolates including laboratory strains. By agar disk diffusion, the results of antibacterial activity of CREO, CAEO, and limonene revealed that these agents had an inhibitory effect against almost all tested bacteria including MRSA and MSSA. CREO exhibited antibacterial activity against both MRSA ATCC 43300 and MSSA ATCC 25923 with the IZDs of $11.3 \pm 1.5 \mathrm{~mm}$ and $11.7 \pm 1.5 \mathrm{~mm}$, respectively. Limonene exhibited antibacterial activity against both MRSA ATCC 43300 and MSSA ATCC 25923 with the IZDs of $12.3 \pm 1.5 \mathrm{~mm}$ and $13.0 \pm 1.7 \mathrm{~mm}$, respectively. On the contrary, CAEO exhibited antibacterial activity against MSSA ATCC 25923 (IZD: $10.3 \pm 3.8 \mathrm{~mm}$ ) but not MRSA ATCC 43300 (IZD: $6.0 \pm 0.0 \mathrm{~mm})$.

For clinical isolates, the IZDs of CREO against MRSA and MSSA were $11.7 \pm 3.1 \mathrm{~mm}$ and $12.0 \pm 2.5 \mathrm{~mm}$, respectively. The IZDs of CAEO were $11.6 \pm 3.9 \mathrm{~mm}$ and $10.0 \pm 2.9 \mathrm{~mm}$ for MRSA and MSSA, while those of limonene were $14.5 \pm 3.9 \mathrm{~mm}$ and $14.4 \pm 2.9 \mathrm{~mm}$ for MRSA and MSSA, respectively. However, 2 clinical isolates, $1 \mathrm{MRSA}$ and 1 MSSA, had no activity (IZD: $6.0 \pm 0.0 \mathrm{~mm}$ ) against CREO, whereas 5 clinical isolates, 3 MRSA and 2 MSSA, had no activity against CAEO. All clinical isolates were inhibited by limonene. By the results of agar disk diffusion, it also demonstrated that CREO inhibited clinically isolated MSSA better than CAEO with a significant difference $(p<0.01)$. 


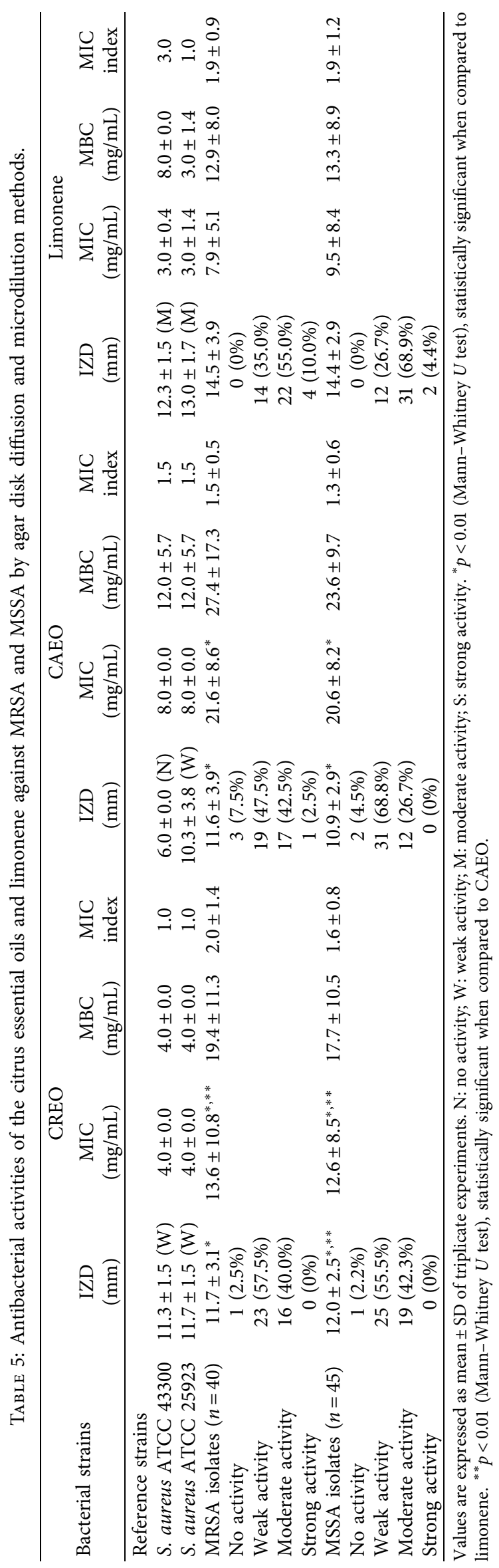


TABLE 6: Combination interaction of the citrus EOs and limonene with gentamicin on MRSA and MSSA by the checkerboard titration method.

\begin{tabular}{|c|c|c|c|c|c|c|c|}
\hline \multirow{2}{*}{ Isolate no. } & \multirow{2}{*}{ Type } & \multirow{2}{*}{ Compounds } & \multicolumn{2}{|c|}{ MIC* } & \multirow{2}{*}{ FIC $^{*}$} & \multirow{2}{*}{ FIC index } & \multirow{2}{*}{ Interaction } \\
\hline & & & Alone & Combination & & & \\
\hline \multirow{6}{*}{ S321 } & \multirow{6}{*}{ MRSA } & CREO & $32.0 \pm 0.0$ & 0.25 & 0.008 & \multirow{2}{*}{0.012} & \multirow{2}{*}{ Synergy } \\
\hline & & Gentamicin & $0.5 \pm 0.0$ & 0.002 & 0.004 & & \\
\hline & & CAEO & $32.0 \pm 0.0$ & 0.25 & 0.008 & \multirow{2}{*}{0.012} & \multirow{2}{*}{ Synergy } \\
\hline & & Gentamicin & $0.5 \pm 0.0$ & 0.002 & 0.004 & & \\
\hline & & Limonene & $24.0 \pm 11.3$ & 0.25 & 0.010 & \multirow{2}{*}{0.012} & \multirow{2}{*}{ Synergy } \\
\hline & & Gentamicin & $0.5 \pm 0.0$ & 0.001 & 0.004 & & \\
\hline \multirow{6}{*}{ S171 } & \multirow{6}{*}{ MRSA } & CREO & $32.0 \pm 0.0$ & 0.25 & 0.008 & \multirow{2}{*}{0.016} & \multirow{2}{*}{ Synergy } \\
\hline & & Gentamicin & $0.25 \pm 0.0$ & 0.002 & 0.008 & & \\
\hline & & CAEO & $32.0 \pm 0.0$ & 0.25 & 0.008 & \multirow{2}{*}{0.016} & \multirow{2}{*}{ Synergy } \\
\hline & & Gentamicin & $0.25 \pm 0.0$ & 0.002 & 0.008 & & \\
\hline & & Limonene & $12.0 \pm 5.7$ & 0.25 & 0.021 & \multirow{2}{*}{0.029} & \multirow{2}{*}{ Synergy } \\
\hline & & Gentamicin & $0.25 \pm 0.0$ & 0.002 & 0.008 & & \\
\hline & & CREO & $12.0 \pm 5.7$ & 4.00 & 0.333 & & \\
\hline & & Gentamicin & $64.0 \pm 0.0$ & 16.00 & 0.250 & 0.583 & Additive \\
\hline & & CAEO & $16.0 \pm 0.0$ & 8.00 & 0.500 & & \\
\hline G44b & MRSA & Gentamicin & $64.0 \pm 0.0$ & 32.00 & 0.250 & 1.000 & Additive \\
\hline & & Limonene & $8.0 \pm 0.0$ & 2.00 & 0.250 & & \\
\hline & & Gentamicin & $64.0 \pm 0.0$ & 8.00 & 0.250 & 0.375 & Synergy \\
\hline & & CREO & $3.0 \pm 1.4$ & 4.00 & 1.333 & & Indifference \\
\hline & & Gentamicin & $256.0 \pm 0.0$ & 0.125 & 0.001 & 1.334 & Indifference \\
\hline & & CAEO & $8.0 \pm 0.0$ & 8.00 & 1.000 & & \\
\hline R569 & MRSA & Gentamicin & $256.0 \pm 0.0$ & 0.25 & 0.001 & 1.001 & Additive \\
\hline & & Limonene & $2.0 \pm 0.0$ & 0.50 & 0.250 & & \\
\hline & & Gentamicin & $256.0 \pm 0.0$ & 0.016 & 0.0005 & 0.250 & Synergy \\
\hline & & CREO & $32.0 \pm 0.0$ & 8.00 & 0.250 & & \\
\hline & & Gentamicin & $128.0 \pm 0.0$ & 1.00 & 0.008 & 0.258 & Synergy \\
\hline & & CAEO & $16.0 \pm 0.0$ & 16.00 & 1.000 & & \\
\hline K201 & MRSA & Gentamicin & $128.0 \pm 0.0$ & 0.50 & 0.008 & 1.004 & Additive \\
\hline & & Limonene & $6.0 \pm 2.8$ & 4.00 & 0.667 & & \\
\hline & & Gentamicin & $128.0 \pm 0.0$ & 0.125 & 0.008 & 0.668 & Additive \\
\hline & & CREO & $6.0 \pm 2.8$ & 4.00 & 0.667 & & \\
\hline & & Gentamicin & $256.0 \pm 0.0$ & 1.00 & 0.004 & $0.6 / 1$ & Additive \\
\hline & & CAEO & $8.0 \pm 0.0$ & 8.00 & 1.000 & & \\
\hline R403 & MSSA & Gentamicin & $256.0 \pm 0.0$ & 1.00 & 0.004 & 1.004 & Additive \\
\hline & & Limonene & $15.0 \pm 0.7$ & 2.00 & 1.333 & & \\
\hline & & Gentamicin & $256.0 \pm 0.0$ & 0.063 & 0.004 & 1.334 & Indifference \\
\hline & & CREO & $24.0 \pm 11.3$ & 4.00 & 0.167 & & \\
\hline & & Gentamicin & $48.0 \pm 22.6$ & 0.063 & 0.001 & 0.168 & Synergy \\
\hline H1025 & MSSA & CAEO & $16.0 \pm 0.0$ & 16.00 & 1.000 & & \\
\hline H1025 & MSSA & Gentamicin & $48.0 \pm 22.6$ & 8.00 & 0.001 & 1.167 & Indifference \\
\hline & & Limonene & $6.0 \pm 2.8$ & 4.00 & 0.667 & & \\
\hline & & Gentamicin & $48.0 \pm 22.6$ & 1.00 & 0.001 & 0.688 & Additıve \\
\hline
\end{tabular}

Values are expressed as mean \pm SD of duplicate experiments. Clinical sources are as follows: S: pus/wound; H: hemoculture; R: sputum; G: urine/stool. * MIC and FIC of CREO, CAEO, and limonene are expressed in $\mathrm{mg} / \mathrm{mL}$, whereas those of gentamicin are expressed in $\mu \mathrm{g} / \mathrm{mL}$.

However, the significant difference was not observed in MRSA isolates $(p>0.05)$. For limonene, the major compound of CREO and CAEO, the IZDs against MRSA and MSSA were significantly higher than those of CREO and CAEO (both $p<0.01$ ). The results indicated that limonene was the highest effective agent following CREO and CAEO.

When the levels of IZD were interpreted according to a previous study [27], it demonstrated various degrees of antibacterial activities of CREO, CAEO, and limonene observed among clinical isolates of $S$. aureus. CREO and CAEO exhibited weak activities against the clinical isolates of MRSA (57.5\% and $47.5 \%$, respectively), followed by moderate activity ( $40.0 \%$ and $42.5 \%$, respectively). Likewise, CREO and CAEO exhibited weak activities against the clinical MSSA isolates (55.5\% and $68.8 \%$, respectively), followed by moderate activity $(42.3 \%$ and $26.7 \%$, respectively). On the contrary, limonene exhibited moderate activities (55.0\% and 68.9\%), followed by weak activity (35.0\% and 26.7\%), against the clinical MRSA and MSSA isolates, respectively. As the expected result, it showed that pure limonene, which is considered as the major composition of CREO and CAEO, efficiently inhibited all tested 
bacteria including MRSA and MSSA, with a moderate activity.

According to the microdilution method, oil control, bacterial control, and diluent control showed that no contamination in the tested oils, growth ability of tested bacteria, and no inhibitory effect occurred by the diluent, respectively. In this study, the results found that MRSA ATCC 43300 and MSSA ATCC 25923 were inhibited by CREO with MIC equal to $4.0 \pm 0.0 \mathrm{mg} / \mathrm{mL}$. For clinical isolates, CREO inhibited MRSA (MIC: $13.6 \pm 10.8 \mathrm{mg} / \mathrm{mL}$ ) and MSSA (MIC: $12.6 \pm 8.5 \mathrm{mg} / \mathrm{mL}$ ) with no significant difference $(p>0.05)$. For another EO, the MIC values of CAEO demonstrated antibacterial activities against 2 ATCC strains lower than those of CREO. It inhibited MRSA ATCC 43300 and MSSA ATCC 25923 with MIC equal to $8.0 \pm 0.0 \mathrm{mg} / \mathrm{mL}$. In the same manner as CREO, CAEO also inhibited clinically isolated MRSA and MSSA (MIC: $21.6 \pm 8.6 \mathrm{mg} / \mathrm{mL}$ and $20.6 \pm 8.2 \mathrm{mg} / \mathrm{mL}$, respectively) with no significant difference $(p>0.05)$. However, the minimum concentration of CREO which inhibited bacterial growth was lower than that of CAEO with a significant difference $(p<0.01)$. It demonstrated that CREO inhibited clinically isolated S. aureus better than CAEO. For limonene, the MIC values demonstrated that it inhibited MRSA ATCC 43300 and MSSA ATCC 25923 ranging from 2.0 to $4.0 \mathrm{mg} / \mathrm{mL}$. It also inhibited clinically isolated MRSA (MIC: $7.9 \pm 5.1 \mathrm{mg} / \mathrm{mL}$ ) and MSSA (MIC: $9.5 \pm 8.4 \mathrm{mg} / \mathrm{mL}$ ) with no significant difference $(p>0.05)$. In addition, the MIC values of limonene against MRSA and MSSA isolates were significantly different when compared to those of CAEO $(p<0.01)$. Like the results of agar disk diffusion, the results of MIC indicated that antibacterial activities toward all tested bacteria were sorted in the descending order as follows: limonene, CREO, and CAEO. Regarding MIC indexes, the citrus EOs and limonene tend to be acting as bactericidal agents towards MRSA and MSSA isolates (MIC index: 1.3-2.0).

\subsection{Synergistic Activities of Gentamicin Combined with Citrus} EOs and Limonene. The combination interaction of gentamicin, which is a standard antibiotic against $S$. aureus, combined with citrus EOs or limonene is presented in Table 6. In this study, the synergistic effect was evaluated against 7 clinical isolates containing 5 isolates of MRSA and 2 isolates of MSSA using the checkerboard titration assay. The results demonstrated that gentamicin in combination with CREO showed synergistic interaction (FICI: $0.012-0.258)$ among the most MRSA $(3 / 5,60.0 \%)$ and MSSA isolates $(1 / 2,50.0 \%)$. On the contrary, gentamicin in combination with CAEO showed synergistic interaction (FICI: $0.012-0.016)$ in only 2 isolates of MRSA $(2 / 5,40.0 \%)$ and none in MSSA isolates $(0 / 2,0.0 \%)$. Likewise, gentamicin in combination with limonene showed synergistic interaction (FICI: 0.012-0.375) in almost all MRSA isolates $(4 / 5,80.0 \%)$, but none in MSSA isolates $(0 / 2,0.0 \%)$. However, no antagonistic effect was observed in the combination of gentamicin with CREO, CAEO, or limonene. These findings revealed the synergistic effect of gentamicin with the citrus EOs or limonene on clinical isolates of $S$. aureus, especially MRSA.

\section{Discussion}

The fruit peels of Citrus spp. are byproducts remained in manufacturing processes of several products such as orange juice and lime juice. The development of these byproducts should be approved to increase their worth. One advantage of the fruit peels is that some can be developed as natural antibiotics. Antibacterial activity of the Citrus spp. on a panel of clinical isolates of the resistant and susceptible strains of $S$. aureus has not been reported. This study was therefore interested to apply EOs extracted from the fruit peels of C. reticulata (CREO) and C. aurantifolia (CAEO) to fight against the clinical strains of resistant and susceptible S. aureus, with our expectation to increase the economic value of their byproducts and reduce the emerging of antibiotic-resistant S. aureus. Additionally, this study determined the antibacterial activities of these two citrus essential oils, CREO and CAEO, and their major components in both single effect and combination interaction with gentamicin.

The extraction yields of CREO (0.48\%) and CAEO $(0.30 \%)$ were in similar ranges of amount with the previous studies $(0.22-0.57 \%)[13,14]$. However, this is difficult to make a comparison since there are many variation factors influenced to the yields of EOs such as climate, geographic distribution, genetics of the plant, the part of the plant used, the degree of freshness, the drying period, and the extraction method [31]. The present study showed that CREO and $\mathrm{CAEO}$ are rich in monoterpenes with the major component being D-limonene (72.53\% in CREO and 62.95\% in CAEO). This is similar to the previous study review that mentioned the contents of limonene in CREO and CAEO of 67.0 to $86.0 \%[7,11,14,20,32]$. However, the contents of limonene found in CREO and CAEO in this study were higher than previous studies that reported 29.3 to $58.9 \%$ of limonene $[16,17,33]$. In fact, the chemical compositions of EOs vary depending on origin, genetic background, season, climate, age, ripening stage, and method of extraction [10].

This study used a panel of clinical Gram-positive strains with different susceptibility profiles to evaluate antibacterial activities of citrus EOs. The chosen bacterial strain was S. aureus, including MSSA, MRSA, and MDR phenotypes. It showed that $77.5 \%$ of MRSA was MDR. In addition, almost all MRSA were less susceptible than MSSA in various antibiotics such as ampicillin ( $\beta$-lactam), amoxicillin ( $\beta$-lactam), ciprofloxacin (fluoroquinolone), erythromycin (macrolide), and clindamycin (lincosamide). Therefore, these antibiotics could not be used for the treatment of MRSA infections. Fortunately, some MRSA were still susceptible to gentamicin (25/40), and vancomycin-resistant isolates were not observed in this study. However, the emergence of vancomycin-resistant $S$. aureus (VRSA) has been reported. The prevalence of VRSA was $1.2 \%$ in Asia, $1.1 \%$ in Europe, 3.6\% in America, and 2.5\% in Africa [34]. The prevalence might be increased in the future. To reduce the usage of vancomycin, the combinations of EOs and limonene with gentamicin were evaluated.

In this study, it appeared that CREO, which contained $72.53 \%$ of limonene, had higher effectiveness against MRSA 
and MSSA strains than that observed in CAEO, which contained $62.95 \%$ of limonene; in addition, purified limonene (97\%) also showed a promising effect. In addition, a higher concentration of limonene in CREO leads to a better synergistic effect than that of CAEO. Based on these observations, it indicated that limonene had an influence on the antibacterial potential of EOs. This is in agreement to previous reports $[16,20,35-37]$ but in contrast to some other studies $[14,31,38]$. The antibacterial mechanism of citrus EOs has been described by a previous study. A possible mechanism was cell wall disruption by citrus EOs on MRSA [39]. This study firstly demonstrated the synergistic effects of CREO, CAEO, and limonene with gentamicin on clinical isolates of MRSA and MSSA. This study proposed that the antibacterial activity of the citrus EOs could attribute to their lipophilicity property and the synergy between their major monoterpene hydrocarbons, limonene, and other minor components such as linalool which has been considered to be an antibacterial agent [37]. The mode of action of EOs could be due to the diffusion and accumulation of the oil in the bacterial cell membrane and then increasing cell membrane permeability, leading to cell lysis and leakage of intracellular components. In addition, the disturbance of the cell membrane may disturb vital processes such as energy conversion, nutrient processing, the synthesis of structural macromolecules, and the secretion of growth regulators $[16,20]$. In addition, limonene is believed to accumulate in the bacterial cell membrane and cause the loss of membrane integrity, dissipation of the proton motive force, inhibition of respiration, and ion transport processes $[7,37,40]$. In fact, there are several gentamicin-resistant mechanisms. One resistant mechanism is reducing uptake or decreased cell permeability. The proposed mechanism of synergism is bacterial membrane disruption by the EOs, leading to easier diffusion of gentamicin, an aminoglycoside, across the bacterial membrane. Consequently, this aminoglycoside is able to inhibit bacterial protein synthesis by binding to the $30 \mathrm{~S}$ ribosomal subunit.

Both CREO and CAEO had an inhibitory effect towards MRSA and MSSA isolated from various types of clinical specimens including skin, bloodstream, respiratory, and urinary tracts (data not shown). It implied that these EOs can be applied as antibacterial agents in several products, such as handwashes and nasal or oral sprays, as well as in several routes of exposure via topical, inhalation, or oral routes. The citrus EOs have been classified as Generally Recognized As Safe (GRAS) [41]. Aumeeruddy-Elalf et al. reported that hydrodistilled essential oils from Citrus spp. fruit peels (C. reticulata) have no cytotoxicity to human cells [12]. Lime and mandarin essential oils up to $100 \%$ had no irritating and sensitizing effects on humans. Acute dermal $\mathrm{LD}_{50}$ of lime EO in rabbits and that oral $\mathrm{LD}_{50}$ in rats were equal to $>5 \mathrm{~g} / \mathrm{kg}$ [10]. By this strategy, it can minimize the adverse effects of gentamicin in view of reducing the treatment dosage to the resistant bacteria, including reducing treatment costs and providing a therapeutic option with greater antimicrobial potential. Further studies on the precise mode of action, therapeutic dosage, tolerability, and safety of the EOs are necessary to provide therapeutic usage of EOs and in combination with antibiotics.

\section{Conclusions}

This study demonstrated that EOs extracted from C. reticulata and C. aurantifolia exhibited antibacterial activities against clinical isolates of $S$. aureus, both MRSA and MSSA. The synergistic effects of EOs with gentamicin toward the clinical isolates of MRSA were also revealed. The usage of these EOs would directly inhibit both susceptible and resistant bacteria and indirectly delay the emergence of bacterial resistance, hence the potential of plant-derived antibacterial agents to be used as a complementary therapy with the established antibiotics that would allow for dose reduction of the antibiotic, thereby delaying and reducing the emergence of antibiotic-resistant strains as well as minimizing the possible side effects.

\section{Data Availability}

All data are included within the manuscript as tables and figures.

\section{Ethical Approval}

This study, including the collection of clinical bacterial isolates, was approved by the Research Ethics Committee of the Faculty of Medicine, Vajira Hospital, Navamindradhiraj University, Thailand (no. COE011/2019), and the Research Ethics Committee of Rangsit University, Thailand (no. RSUERB2019-027).

\section{Conflicts of Interest}

The authors declare that they have no conflicts of interest.

\section{Acknowledgments}

The authors would like to thank the Faculty of Medical Technology, Rangsit University, Thailand, for providing research facilities and staff in the Division of Microbiology, Department of Central Laboratory and Blood Bank, Faculty of Medicine, Vajira Hospital, Navamindradhiraj University, Thailand, for facilitating collection of the bacterial isolates in this study. Also, they gratefully thank Asst. Prof. Matthew Tucker for reviewing this manuscript. This study was funded by the Research Institute of Rangsit University, Thailand (Grant no. 1-2019).

\section{References}

[1] K. M. Craft, J. M. Nguyen, L. J. Berg, and S. D. Townsend, "Methicillin-resistant Staphylococcus aureus (MRSA): antibiotic-resistance and the biofilm phenotype," Medicinal Chemistry Communication, vol. 10, no. 8, pp. 1231-1241, 2019.

[2] S. Lakhundi and K. Zhang, "Methicillin-resistant Staphylococcus aureus: molecular characterization, evolution, and epidemiology," Clinical Microbiology Reviews, vol. 31, no. 4, p. 103, Article ID e00020-18, 2018.

[3] Y. Guo, G. Song, M. Sun, J. Wang, and Y. Wang, "Prevalence and therapies of antibiotic-resistance in Staphylococcus aureus," Frontiers in Cellular and Infection Microbiology, vol. 10, p. 107, 2020. 
[4] S. Patel, C. V. Preuss, and F. Bernice, "Vancomycin," in StatPearls [Internet]StatPearls Publishing, Treasure Island, FL, USA, 2021.

[5] S. Md Othman, M. Hassan, L. Nahar, N. Basar, S. Jamil, and S. Sarker, "Essential oils from the Malaysian citrus (rutaceae) medicinal plants," Medicines, vol. 3, no. 2, p. 13, 2016.

[6] "Office of Agricultural Economics, Thailand," 2019, http:// www.oae.go.th/view/1/Information/EN-US.

[7] S. Frassinetti, L. Caltavuturo, M. Cini, and C. M. Della Croce, "Antibacterial and antioxidant activity of essential oils from Citrus spp." Journal of Essential Oil Research, vol. 23, pp. 27-31, 2011.

[8] J. H. Lee, S. Cho, and H. D. Paik, "Investigation on antibacterial and antioxidant activities, phenolic and flavonoid contents of some Thai edible plants as an alternative for antibiotics," Asian-Australasian Journal of Animal Sciences, vol. 27, no. 10, pp. 1461-1468, 2014.

[9] R. A. W. Ernawita, J. Hesse, U. C. Hipler, P. Elsner, and V. Böhm, "In vitro lipophilic antioxidant capacity, antidiabetic and antibacterial activity of Citrus fruits extracts from Aceh, Indonesia," Antioxidants, vol. 6, no. 1, p. 11, 2017.

[10] N. S. Dosoky and W. N. Setzer, "Review: biological activities and safety of Citrus spp. essential oils," International Journal of Molecular Sciences, vol. 19, p. 1966, 2018.

[11] X. Lin, S. Cao, J. Sun, D. Lu, B. Zhong, and J. Chun, "The chemical compositions, and antibacterial and antioxidant activities of four types of Citrus essential oils," Molecules, vol. 26, no. 11, p. 3412, 2021.

[12] Z. Aumeeruddy-Elalf, I. S. Ismaël, M. Hosenally, G. Zengin, and M. F. Mahomoodally, "Essential oils from tropical medicinal herbs and food plants inhibit biofilm formation in vitro and are non-cytotoxic to human cells," 3 Biotech, vol. 8, no. 9, p. 395, 2018.

[13] S. Chanthaphon, S. Chanthachum, and T. Hongpattarakere, "Antimicrobial activities of essential oils and crude extracts from tropical Citrus spp. against food-related microorganisms," Songklanakarin Journal of Science and Technology, vol. 30, pp. 125-131, 2008.

[14] S. Bourgou, F. Z. Rahali, I. Ourghemmi, and M. Saïdani Tounsi, "Changes of peel essential oil composition of four Tunisian citrus during fruit maturation," The Scientific World Journal, vol. 2012, Article ID 528593, 10 pages, 2012.

[15] S. Saeb, M. Amin, R. S. Gooybari, and N. Aghel, "Evaluation of antibacterial activities of Citrus limon, Citrus reticulata, and Citrus grandis against pathogenic bacteria," International Journal of Enteric Pathogens, vol. 4, no. 4, 5 pages, Article ID e37103, 2016.

[16] H. Soetjipto, "Antibacterial properties of essential oil in some Indonesian herbs," Potential of Essential Oils, H. El-Shemy, eds, IntechOpen, London, UK, 2018.

[17] H. S. Hou, E. M. Bonku, and R. Zhai, "Extraction of essential oil from Citrus reticulate Blanco peel and its antibacterial activity against Cutibacterium acnes (formerly Propionibacterium acnes)," Heliyon, vol. 5, no. 12, Article ID e02947, 2019.

[18] A. Shakya, B. Luitel, P. Kumari, R. Devkota, P. R. Dahal, and R. Chaudhary, "Comparative study of antibacterial activity of juice and peel extract of Citrus fruits," Tribhuvan University Journal of Microbiology, vol. 6, no. 1, pp. 82-88, 2019.

[19] S. Afroja, F. N. Falgunee, M. M. Jahan, K. M. Akanda, S. Mehjabin, and G. M. M. Parvez, "Antibacterial activity of different Citrus fruits," Specialty Journal of Medical research and Health Science, vol. 2, no. 1, pp. 25-32, 2017.
[20] X. Song, T. Liu, L. Wang, L. Liu, X. Li, and X. Wu, "Antibacterial effects and mechanism of Mandarin (Citrus reticulata L.) essential oil against Staphylococcus aureus," Molecules, vol. 25, no. 21, p. 4956, 2020.

[21] N. Bektaš, B. Gazić, and M. Avdić, "Antibacterial activity of citrus juices and apple vinegar on Gram positive and Gram negative bacteria," International Journal of Innovative Science and Research Technology, vol. 4, no. 11, pp. 837-841, 2019.

[22] A. T. Vong, H. W. Chong, and V. Lim, "Preliminary study of the potential extracts from selected plants to improve surface cleaning," Plants, vol. 7, no. 1, p. 17, 2018.

[23] S. Chao, G. Young, C. Oberg, and K. Nakaoka, "Inhibition of methicillin-resistant Staphylococcus aureus (MRSA) by essential oils," Flavour and Fragrance Journal, vol. 23, pp. 444-449, 2008.

[24] A. R. Pintong, J. Ruangsittichai, and S. Ampawong, "Efficacy of Ageratum conyzoides extracts against Giardia duodenalis trophozoites: an experimental study," BMC Complementary Medicine and Therapies, vol. 20, no. 1, 9 pages, Article ID 25178298, 2020.

[25] Clinical and Laboratory Standards Institute (CLSI), Performance Standards for Antimicrobial Susceptibility Testing, CLSI, Wayne, PA, USA, 29th edition, 2019.

[26] A. Sreepian, P. M. Sreepian, C. Chanthong, T. Mingkhwancheep, and P. Prathit, "Antibacterial activity of essential oil extracted from Citrus hystrix (kaffir lime) peels: an in vitro study," Tropical Biomedicine, vol. 36, no. 2, pp. 531-541, 2019.

[27] F. Lv, H. Liang, Q. Yuan, and C. Li, "In vitro antimicrobial effects and mechanism of action of selected plant essential oil combinations against four food-related microorganisms," Food Research International, vol. 44, no. 9, pp. 3057-3064, 2011.

[28] M. Elshikh, S. Ahmed, and S. Funston, "Resazurin-based 96well plate microdilution method for the determination of minimum inhibitory concentration of biosurfactants," Biotechnology Letters, vol. 38, no. 6, pp. 1015-1019, 2016.

[29] D. Gatsing, V. Tchakoute, and D. Ngamga, "In vitro antibacterial activity of Crinum purpurascens herb leaf extract against the Salmonella species causing typhoid fever and its toxicological evaluation," Iranian Journal of Medical Sciences, vol. 34, pp. 126-136, 2009.

[30] S. Mulyaningsih, F. Sporer, S. Zimmermann, J. Reichling, and M. Wink, "Synergistic properties of the terpenoids aromadendrene and 1,8-cineole from the essential oil of Eucalyptus globulus against antibiotic-susceptible and antibiotic-resistant pathogens," Phytomedicine, vol. 17, pp. 1061-1066, 2010.

[31] D. Djenane, "Chemical profile, antibacterial and antioxidant activity of Algerian citrus essential oils and their application in Sardina pilchardus," Foods, vol. 4, pp. 208-228, 2015.

[32] R. S. Lemes, C. C. F. Alves, and E. B. B. Estevam, "Chemical composition and antibacterial activity of essential oils from Citrus aurantifolia leaves and fruit peel against oral pathogenic bacteria," Academia Brasileira De Ciencias, vol. 90, no. 2, pp. 1285-1292, 2018.

[33] S. K. Fagodia, H. P. Singh, D. R. Batish, and R. K. Kohli, "Phytotoxicity and cytotoxicity of Citrus aurantifolia essential oil and its major constituents: limonene and citral," Industrial Crops and Products, vol. 108, pp. 708-715, 2017.

[34] A. Shariati, M. Dadashi, and M. T. Moghadam, "Global prevalence and distribution of vancomycin resistant, vancomycin intermediate and heterogeneously vancomycin intermediate Staphylococcus aureus clinical isolates: a systematic 
review and meta-analysis," Scientific Reports, vol. 10, p. 16, Article ID 12689, 2020.

[35] A. B. Hsouna, N. Hamdi, N. B. Halima, and S. Abdelkafi, "Characterization of essential oil from Citrus aurantium L. flowers: antimicrobial and antioxidant activities," Journal of Oleo Science, vol. 62, no. 10, pp. 763-772, 2013.

[36] D. Teneva, R. Denkova-Kostova, and B. Goranov, "Chemical composition, antioxidant activity and antimicrobial activity of essential oil from Citrus aurantium L zest against some pathogenic microorganisms," Zeitschrift für Naturforschung C, vol. 74, no. 5-6, pp. 105-111, 2019.

[37] T. T. K. Ngan, O. B. Nguyen, N. V. Muoi, T. T. Truc, and V. T. N. My, "Chemical composition and antibacterial activity of orange (Citrus sinensis) essential oils obtained by hydrodistillation and solvent free microwave extraction," IOP Conference Series: Materials Science and Engineering, vol. 991, 2020.

[38] M. Soković, J. Glamočlija, P. D. Marin, D. Brkić, and L. J. van Griensven, "Antibacterial effects of the essential oils of commonly consumed medicinal herbs using an in vitro model," Molecules, vol. 15, no. 11, pp. 7532-7546, 2010.

[39] A. Muthaiyan, E. M. Martin, S. Natesan, P. G. Crandall, B. J. Wilkinson, and S. C. Ricke, "Antimicrobial effect and mode of action of terpeneless cold pressed valencia orange essential oil on methicillin-resistant Staphylococcus aureus," Journal of Applied Microbiology, vol. 112, no. 5, pp. 1020-1033, 2012.

[40] B. Chueca, R. Pagán, and D. García-Gonzalo, "Differential mechanism of Escherichia coli Inactivation by (+)-limonene as a function of cell physiological state and drug's concentration," PLoS One, vol. 9, no. 4, p. 7, Article ID e94072, 2014.

[41] R. Tisserand and R. Young, Essential Oil Safety, Elsevier, New York, NY, USA, 2nd edition, 2014. 